\title{
Maßgeschneiderte Sanierungsverfahren für die Tunnelentwässerung - DrainRepair
}

\author{
Florian Arbeiter ${ }^{1}$, Tobias Schachinger ${ }^{2}$, Robert Wenighofer ${ }^{3}$, Giesbert Rieß ${ }^{4}$, Michael Steiner ${ }^{5}$ Andreas Schoen ${ }^{2}$, \\ Michael Griebaum ${ }^{6}$, Viktor Laufer ${ }^{7}$ und Robert Galler ${ }^{3}$
}

\author{
${ }^{1}$ Lehrstuhl für Werkstoffkunde und Prüfung der Kunststoffe, Montanuniversität Leoben, Leoben, Österreich \\ ${ }^{2}$ ÖBB Infrastruktur AG, Wien, Österreich \\ ${ }^{3}$ Lehrstuhl für Subsurface Engineering, Montanuniversität Leoben, Leoben, Österreich \\ ${ }^{4}$ Lehrstuhl für Chemie der Kunststoffe, Montanuniversität Leoben, Leoben, Österreich \\ ${ }^{5}$ ASFINAG, Wien, Österreich \\ ${ }^{6} \mathrm{RTi}$ Austria GmbH, Pucking, Österreich \\ ${ }^{7}$ NordiTube Technologies SE, Liège, Belgien
}

Eingegangen 16. Oktober 2021; angenommen 3. November 2021; online publiziert 30. November 2021

\begin{abstract}
Zusammenfassung: Drainagesysteme dienen in druckwasserentlasteten Tunnelbauwerken der permanenten konzentrierten Ableitung des anfallenden Grundwassers. Zur Sicherstellung der Funktionstüchtigkeit der Bauwerke müssen diese Drainagen in regelmäßigen Abständen gewartet und gereinigt werden. Vor allem bei schwer lösbaren Versinterungen kann es durch intensive Reinigung auch zu Schäden an den Drainage-Rohren selbst kommen. Je nach auftretendem Schadensbild können diese defekten Rohre im schlimmsten Fall sogar eine Bedrohung für das gesamte Bauwerk darstellen. Aus diesem Grund ist es unerlässlich, beschädigte Drainage-Rohre zeitnah instand zu setzen. Aus dem Bereich der kommunalen Wasserwirtschaft und dem Kanalwesen sind einige Verfahren, die zur grabenlosen Sanierung von Rohren standardmäßig eingesetzt werden können, bekannt. Das Problem bestehender Verfahren ist, dass diese die spezifischen tunnelbaulichen Aspekte, wie die benötigte Drainagewirkung, eingeschränkte Zutrittsmöglichkeiten über Putzschächte, keine komplette Absperrmöglichkeit des Drainagewassers, intensivere Reinigungsarbeiten etc. bis dato meist nicht berücksichtigen.
\end{abstract}

Schlüsselwörter: Tunnelbau, Drainagerohre, Sanierung, Grabenlos

Dr. F. Arbeiter $(\bowtie)$

Lehrstuhl für Werkstoffkunde und Prüfung der Kunststoffe, Montanuniversität Leoben,

Leoben, Österreich

florian.arbeiter@unileoben.ac.at
Tailored Rehabilitation for Tunnel Drainage Pipes-DrainRepair

Abstract: Drainage systems in pressure-relieved tunnels are used for the permanent concentrated drainage of ground water. In order to ensure that the tunnel structures function properly, these drainage systems must be maintained and cleaned at regular intervals. Intensive cleaning can also cause damage to the drainage pipes themselves, especially in the case of hard precipitations in the pipes that are difficult to remove. Depending on the type of damage, these defective pipes can even pose a threat to the entire structure in the worst case. For this reason, it is essential to repair damaged drainage pipes promptly.

A number of methods that can be used for trenchless pipe rehabilitation are known from the field of municipal water management and sewage. The problem with existing methods is that they usually do not consider the specific tunnel construction aspects, such as the required drainage effect, limited access via cleaning shafts, no complete shutoff of the drainage water, more intensive and mechanical cleaning work compared to municipal water pipes, and more.

Keywords: Tunnelling, Drainage pipes, Rehabilitation, Trenchless

\section{Tunnelabdichtungs- und Tunnelentwässe- rungskonzepte}

Die Ausbildung eines Tunnelquerschnittes wird maßgebend von den umgebenden Grundwasserverhältnissen 
bestimmt. Liegt ein Tunnel unter dem Grundwasserspiegel, ist entweder eine Auslegung des Bauwerkes auf den Wasserdruck (druckwasserhaltende Ausbildung) oder ein Abbau des umgebenden Wasserdrucks (druckwasserentlastete Ausbildung) erforderlich. Neben der technischen Machbarkeit sind Kriterien für die Auswahl u.a. die Errichtungskosten, Umweltaspekte (insbesondere der potenzielle Eingriff in den Wasserhaushalt) und die späteren Instandhaltungskosten.

Nachfolgend werden die unterschiedlichen Ausbildungen kurz beschrieben.

\subsection{Druckwasserentlastete Ausbildung eines Tunnelbauwerks}

Dabei wird der Wasserdruck auf Bestandsdauer durch Drainagen, die als Freispiegelentwässerung wirken, abgebaut. Im Regelfall ist nur eine Abdichtung gegen nicht drückendes Wasser, eine sogenannte Regenschirmabdichtung, im Gewölbe erforderlich. Da das Tunnelbauwerk aus statischer Hinsicht nicht auf den Wasserdruck auszulegen ist, ist aus diesem Lastfall resultierend üblicherweise keine Bewehrung erforderlich. Ein aus Instandhaltungssicht wesentlicher Bauteil sind die Drainagen. Diese wurden früher als eckige gemauerte Kanäle oder Gräben in der Sohle, heutzutage als Drainagerohre im seitlichen („Ulmendrainagen“) bzw. unteren Bereich („Sohldrainagen“) des Tunnelbauwerks ausgebildet.

Aufgrund geogener Gründe und/oder der eingesetzten Baumaterialien kommt es in den Drainagen oftmals zu Ausfällungen [1-3]. Diese meist aus Kalzit bestehenden Versinterungen müssen zur Sicherstellung der Funktionstüchtigkeit der Bauwerke in regelmäßigen Abständen aus den Drainagen entfernt werden. Dazu ist es vorab nötig, die Drainagen zu inspizieren und mittels hydraulischer oder mechanischer Reinigungsverfahren von Versinterungen zu befreien. Aufgrund der schwierigen Bedingungen im Tunnelbauwerk, die oftmals auch eine Sperre der Tunnel für die Dauer der Reinigung bedeuten, führt dies bei allen Infrastrukturbetreibern zu hohen Betriebseinschränkungen und Kosten in der Instandhaltung.

Zahlreiche Tunnelbauwerke wurden und werden druckwasserentlastet ausgeführt. In der Vergangenheit war es aufgrund der Bauweise (beispielsweise bei gemauerten Tunnelbauwerken) oft nicht anders möglich. Bei modernen Tunnelbauwerken, die mehr als $30 \mathrm{~m}$ unter dem natürlichen Grundwasserspiegel liegen, sind die Drainagen oftmals die einzige Möglichkeit, ein Bauwerk aus technischer Sicht zu errichten.

Aufgrund des hohen Instandhaltungsaufwandes für die Tunneldrainagen wird jedoch in Mitteleuropa mittlerweile versucht, vor allem seichtere Tunnelbauwerke auf den Wasserdruck auszulegen.

\subsection{Druckwasserhaltende Ausbildung eines Tunnelbauwerks}

Bei dieser Ausbildung wird das Tunnelbauwerk durch eine entsprechend dichte Ausführung auf den Wasserdruck des umgebenden Grundwassers ausgelegt. Die dichte Ausführung kann entweder durch folienförmige Abdichtungssysteme aus Kunststoff oder durch eine dichte, üblicherweise bewehrte, Ausführung der Betonbauteile erreicht werden.

Gründe für diese Ausbildung sind beispielsweise die Verhinderung des dauerhaften Eingriffs in den Wasserhaushalt (z. B. oft erforderlich bei innerstädtischen Tunnelbauwerken) oder ein entsprechend seichter Grundwasserspiegel, sodass auf Drainagen verzichtet werden kann und somit der Instandhaltungsaufwand in der Betriebsphase des Tunnelbauwerks geringer ist.

Demgegenüber stehen jedoch bedeutend höhere Investitionskosten, da der bauliche Aufwand für die Errichtung eines druckwasserhaltenden Bauwerk bedeutend höher ist als für ein druckwasserentlastetes.

\subsection{Druckgeregelte Tunnel}

Einen theoretischen Sonderfall stellt eine druckgeregelte Entwässerung dar. Dabei sollte der Wasserdruck nur zum Teil abgebaut werden, indem die abzuleitende Wassermenge durch einen künstlich erzeugten Rückstau am Auslauf des Entwässerungssystems reduziert wird. Die Belastung von Abdichtung und Auskleidung ist abhängig vom verbleibenden Wasserdruck. Die abzuleitende Wassermenge wird umso mehr reduziert, je höher der Wasserdruck gewählt wird. Das druckgeregelte Tunnelentwässerungssystem stellt damit eine Alternative zur Tunneldrainage als Freispiegelleitung dar. Es handelt sich dabei um ein ständig wassergefülltes Leitungssystem, bei dem der Kontakt des Wassers mit der Luft erst an der Austrittsöffnung am Portal stattfindet. Dies sollte die Menge an anfallender Versinterung reduzieren. Aufgrund des extrem hohen Instandhaltungsaufwandes des Druckentlastungssystems wurde nach Wissen der Autoren ein derartiges System bisher nicht dauerhaft in einem Tunnelbauwerk angewendet.

\section{Probleme mit Drainagen}

Wie in Abschnitt 1 und [4,5] bereits angeführt, dienen Drainagen in druckwasserentlasteten Tunnelbauwerken der permanenten konzentrierten Ableitung des anfallenden Grundwassers. Dafür wurden ab der zweiten Hälfte des 20. Jahrhunderts Rohre aus verschiedenen Materialien mit Drainage- und Wasserableitefunktion verwendet. Seit den 90er-Jahren werden ausschließlich Rohre aus thermoplastischen Kunststoffen verwendet. Konkret werden heutzutage gemäß Richtlinie der Österreichischen Vereinigung für Beton- und Bautechnik (ÖVBB) [6] Rohre aus nicht plastifiziertem Polyvinylchlorid (UPVC), Polypropylen (PP) und Polyethylen (PE) zum Einsatz. Bedingt durch die laufende umfangreiche Bautätigkeit werden in den Tunnelbauwerken der ÖBB, der Asfinag und der Bundesländer 
Abb. 1: Beschädigungen der Drainagerohre im Melkertunnel (COBB Infrastruktur AG)
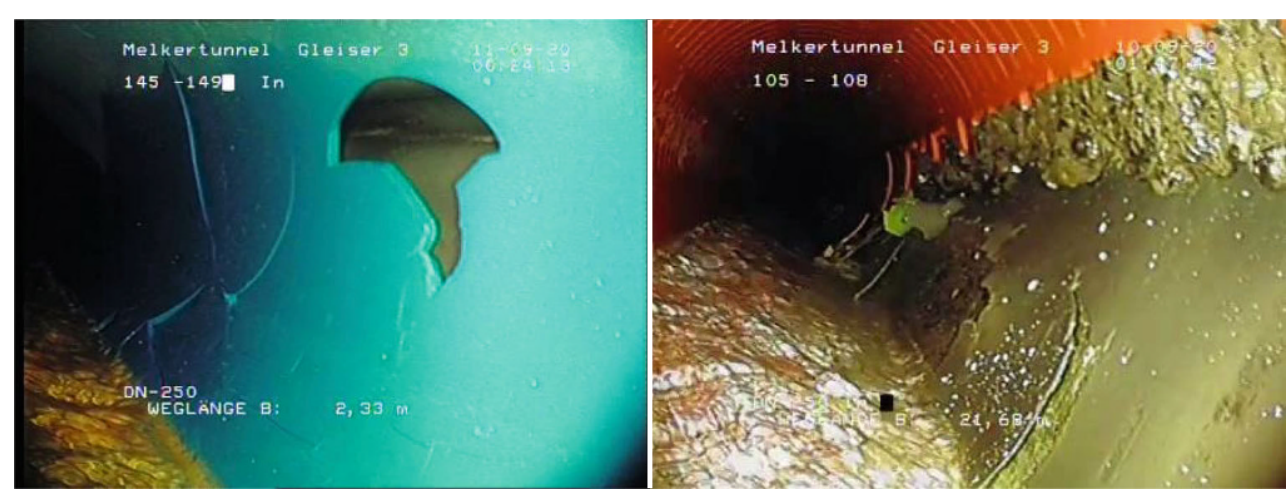

Abb. 2: Beschädigungen der Drainagerohre im Siebergtunnel (৫ÖBB Infrastruktur AG)

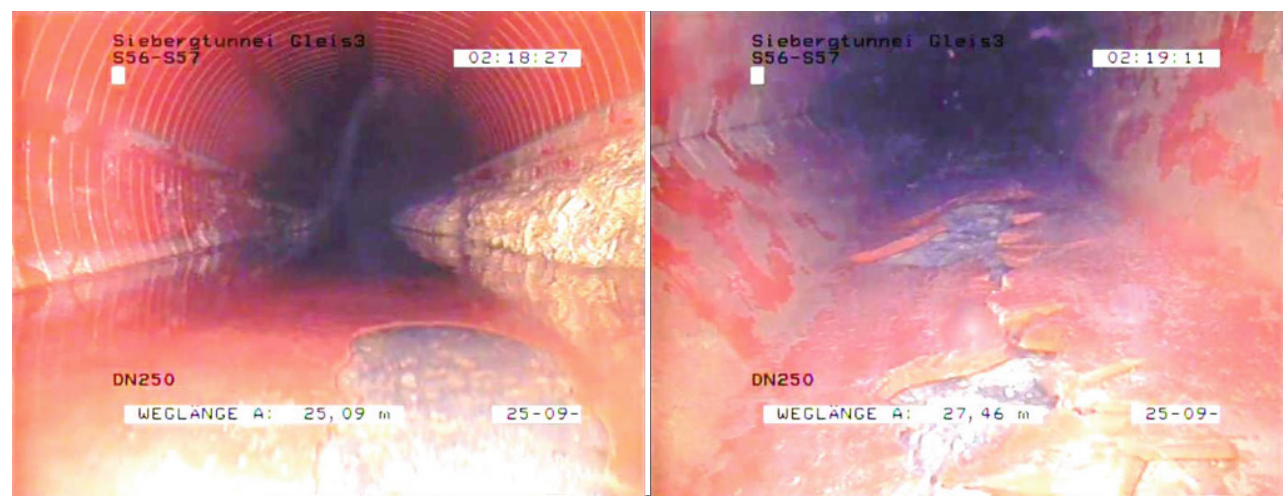

Abb. 3: Beschädigung des tunnel (CRTi Austria) Drainagerohrs im Gleisswand-

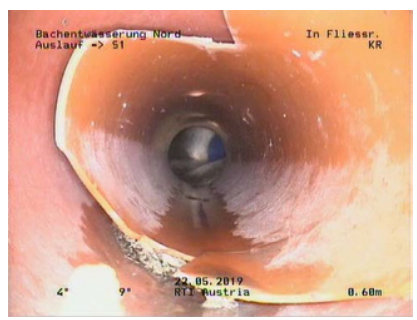

bis hin zu Rohrbrüchen und herausgeschlagenen großen Rohrstücken reichen.

Als Grund für diese Art der Beschädigung bzw. Zerstörung der verwendeten Drainagerohre wird eine Kombination von Ursachen vermutet. Ausgehend von oftmals fehlenden klaren Anforderungsprofilen hinsichtlich der mechanischen Eigenschaften der Drainagerohre, wurden wie oben erwähnt meist relativ dünnwandige geschlitzte Rohre aus uPVC verwendet. Dies ist primär darauf zurückzuführen, dass uPVC, im Vergleich zu PP und PE eine relativ hohe Steifigkeit aufweist und somit mit vergleichsweise wenig Materialeinsatz, und damit verbundenen geringen Herstellungskosten, bereits die in den 90er-Jahren geforderte notwendige statische Tragfähigkeit aufweist. Basierend auf dem heutigen Wissensstand, dürfte jedoch nicht nur die statische Tragfähigkeit, sondern vor allem auch der Widerstand gegenüber schlagender Belastung während der Reinigung essentiell für die Langlebigkeit von Drainagerohren sein [9]. Aufgrund der im Vergleich zu Polyolefinen (PP \& PE) meist höheren Kerbempfindlichkeit von uPVC [9] und den vorhandenen Drainageschlitzen sowie den hohen, schlagartig eingebrachten, mechanischen Belastungen während intensiver Reinigungsvorgänge könnte es somit zum Versagen des Drainagerohres gekommen sein. Es sollte erwähnt werden, dass es alleine bei den ÖBB mehr als $200 \mathrm{~km}$ dieser Drainagerohre gibt, die in der Zukunft das Potenzial für derartige Rohrschäden haben.

Neben dem potenziellen Verlust der Drainagewirkung der Rohre können diese Rohrschäden ebenfalls zu einer erschwerten Instandhaltung (beispielsweise verhakende Spülwerkzeuge) bis zu gar nicht mehr möglichen Instandhaltung (beispielsweise wenn die Sickerpackung ringsum 
Abb. 4: Regelquerschnitt Siebergtunnel mit Markierung der Innenschale sowie des Abdichtungssystems, die bei einer Neuverlegung von Drainagerohren entfernt und wieder Instand-gesetzt werden müssten (C)IGT Geotechnik und Tunnelbau Ziviltechniker $\mathrm{GmbH}$ )

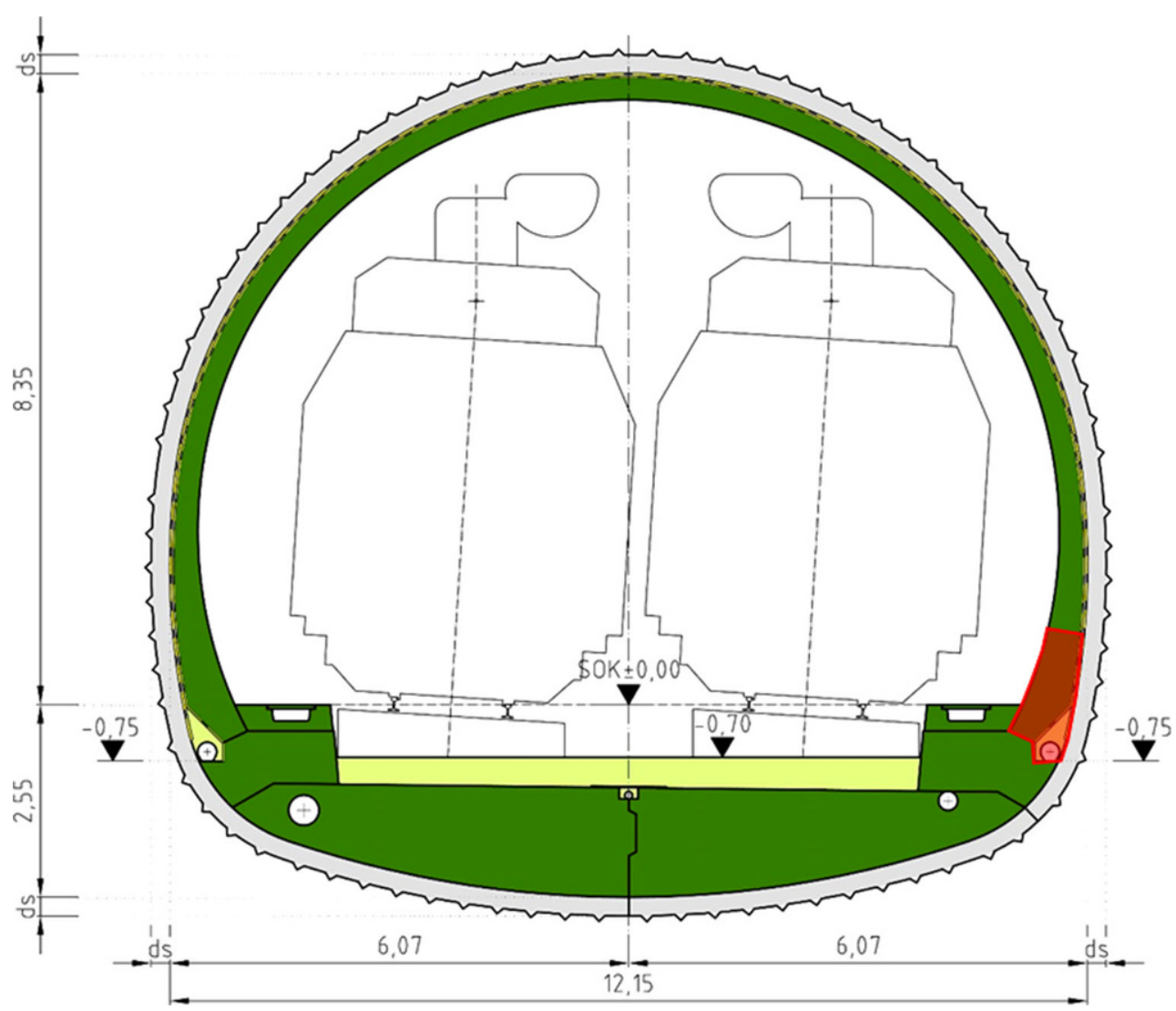

2.1 Randbedingungen und Anforderungen im Tunnelbau

inbricht) führen. In jedem Fall sinkt die Verfügbarkeit und Zuverlässigkeit des Tunnelbauwerkes und es ist unabdingbar, beschädigte Drainagerohre zeitnahe instand zu setzen.

Um das Drainagerohr vollständig durch ein neues Rohr zu ersetzen, müsste bei einem zweischaligen Tunnelbauwerk die Innenschale und das Abdichtungssystem, in Abb. 4 rot markiert, entfernt werden. Dies würde eine Totalsperre des Tunnels und lange und kostenintensive Bauarbeiten bedingen.

Aus diesem Grund sollten Instandsetzungen von Drainagerohren, sofern möglich, prinzipiell mittels grabenloser Verfahren, wie sie im Bereich der kommunalen Wasserwirtschaft und dem Kanalwesen standardmäßig eingesetzt werden, durchgeführt werden. Einen Überblick über derzeit übliche Verfahren bieten die ÖNORM EN 15885 [10] sowie die Homepage der Österreichische Vereinigung für Grabenlosen Leitungsbau [11]. Das Hauptproblem bei der Umsetzung dieser Sanierungsmethoden auf Drainagerohre im Tunnel sind dabei jedoch die speziellen Einschränkungen und Randbedingungen des Tunnelbaus selbst. Zur Verdeutlichung sind einige dieser Einschränkungen nachfolgend näher ausgeführt.

\subsubsection{Randbedingungen}

1. Beschränkte Zugänglichkeit - im Tunnelbauwerk ist die Zugänglichkeit der Drainagerohre auf sogenannte Putzschächte im Abstand von 50 bis ca. $75 \mathrm{~m}$ beschränkt. Sie weisen Abmessungen von zumindest $60 * 140 \mathrm{~cm}$ bei einer Tiefe bis $150 \mathrm{~cm}$ auf. Teilweise befinden sich diese Putzschächte auch in Nischen im Bauwerk. Diese Putzschächte müssen in Form und Lage erhalten bleiben.

2. In österreichischen Tunnelbauwerken gibt es unterschiedliche Geometrien der Drainagerohre, d.h. es gibt runde Rohre und Maul- bzw. Tunnelprofile;

3. Wasserzutritt während der Sanierungsarbeiten - Während des Sanierungsvorganges gibt es keine Absperrmöglichkeit für das zur Drainage von außen aus dem Gebirge zutretende Grundwasser. Dies könnte bei manchen Verfahren unter Umständen die Aushärtung von Harzen negativ beeinflussen.

4. Die Drainagerohre sind in Beton, Drainagebeton oder Drainagekies gebettet. Teilweise gibt es Situationen, bei denen das Rohr im Bereich der Drainageschlitze verbrochen ist und sich ehemals darüber ein nicht zementös gebundener Drainagekies befunden hat. In diesen Fällen ist der Drainagekies in das Rohr eingedrungen und es existiert keine Bettung mehr. Da die Bettung der Drainagerohre meistens aus zementgebundenen Baumateria- 
lien besteht, ist sie nicht verdrängbar, wie dies für einige Sanierungsverfahren erforderlich wäre

\subsubsection{Anforderungen}

1. Kein Verlust der Drainagefunktion - In den meisten Fällen sollte das instandgesetzte Rohr eine Drainagefunktion besitzen. Sämtliche Rohrsanierungsverfahren liefern prinzipiell jedoch Vollrohre ohne Drainagefunktion. Es ist möglich, nachträglich Drainageschlitze mittels Rohrroboter wiederherzustellen, allerdings ist dabei zu prüfen, ob die Herstellung der Drainageschlitze zu einer Verringerung der Materialeigenschaften führt

2. Die Innenschale und die Abdichtungsebene des Tunnelbauwerkes dürfen bei der Sanierung des Drainagerohres nicht beschädigt werden.

3. Nach Möglichkeit soll es bedingt durch die Sanierung zu keiner signifikanten Querschnittsreduzierung kommen.

4. Bei ÖBB-Tunnels beträgt die Nutzungsdauer mittlerweile 150 Jahre. Dies umfasst auch das Drainagesystem. Somit sollten die sanierten Rohre möglichst langlebig sein.

5. Die Eigenschaften des Neurohres sollen nach Möglichkeit den Vorgaben der Richtlinie Tunnelentwässerung entsprechen. Die sanierten Rohre sollen die in Tunnels übliche Reinigungsverfahren widerstehen können (beispielsweise Hochdruckreinigung, oder Vibrationsdüsen).

6. In Übergangsbereichen zwischen Neurohr und Altrohr ( „Stufe“) darf es zu keinen Qualitätseinschränkungen kommen (beispielsweise Ablösung des Neurohrs vom Altrohr nach Hochdruckreinigung).

\subsection{Auswahl geeigneter Methoden}

In Rücksichtnahme auf oben genannte Randbedingungen und Anforderungen wurden die aus dem kommunalen Wasserbau verfügbaren Methoden in einer Literatur-Studie hinsichtlich ihrer Eignung untersucht. Vor allem die Notwendigkeit eines Sanierungsverfahrens, das ohne größere Baugrube auskommt, schränkt den Kreis der infrage kommenden Verfahren stark ein. Ein Verfahren, das auf relativ engen Plätzen gut eingesetzt werden kann, ist jedoch das sogenannte "Cured in Place Pipe“ (CIPP) Verfahren. Dabei wird ein mit Harz-getränkter Inliner in das vorhandene, aber beschädigte Rohr eingeblasen und ausgehärtet. Durch die Möglichkeit der Verwendung verschiedener Liner sowie Harze können auch gezielt Eigenschaften dieser Inliner eingestellt werden. Trotz dieser vielversprechenden Eigenschaften wurde in der Vergangenheit beobachtet, dass es beim Einsatz des CIPP Verfahrens zu Komplikationen kommen kann. Konkret wurden bereits eingebaute Liner bei anschließenden Probespülungen wieder zerstört.

Als Problem wurde bei diesen Sanierungsmaßnahmen erkannt, dass neben einem teils fehlenden Know-Hows auf Auftraggeberseite das Verfahren eingesetzt wurde, ohne auf die tunnelspezifischen Randbedingen und Anforderungen, wie in 2.1.1 und 2.1.2 gelistet, Rücksicht zu nehmen.
Vor allem Punkt 5 der Anforderungen, der die Notwendigkeit hoher mechanischer Eigenschaften betont, sollte in Zukunft stärker in Betracht gezogen werden. Um entsprechendes Know-How für zukünftige Rohrsanierungen in Tunnelbauten zu schaffen, wurde das VIF20 DrainRepair ins Leben gerufen [12].

Ziel des Projekts soll dabei sein, an der Adaptierung ausgewählter grabenloser Verfahren in Hinblick auf die tatsächlichen Randbedingungen im Tunnelbau zu arbeiten. Vor allem in Hinblick auf das CIPP Verfahren wäre der Einsatz Unterwasser härtender sowie hinsichtlich Schlagzähigkeit optimierte Harze eine gute Möglichkeit. Zusätzlich ist es möglich das Material des verwendeten Gewebeschlauchs zu optimieren. Es können auch schlagabsorbierende Innenschichten auf Thermoplast-Basis (Polyethylen, Polypropylen oder thermoplastisches Polyurethan) verwendet werden, um die Zähigkeit des Gesamtverbunds zu erhöhen. Nach Adaptierung des Sanierungsverfahren selbst muss als letzter Schritt ebenfalls noch die zu erwartende Lebensdauer der Instandsetzung beurteilt werden. Daher ist es notwendig, spezifische Einflüsse hinsichtlich mechanischer und chemischer Belastung, die in Tunnelbauwerken vorliegen, in eine Abschätzung miteinzubeziehen [12].

\section{Danksagung}

Die durchgeführten Arbeiten in dieser Veröffentlichung wurden im Rahmen des VIF-2020 Projekts "DrainRepair" (879376) mit Beteiligung der ÖBB-Infrastruktur AG sowie der ASFINAG und des Bundesministeriums für Klimaschutz, Umwelt, Energie, Mobilität, Innovation und Technologie. Weiterer Dank der Firma IGT Geotechnik und Tunnelbau Ziviltechniker GmbH für die Erlaubnis zur Verwendung von Abb. 4.

Funding. Open access funding provided by Montanuniversität Leoben.

Open Access Dieser Artikel wird unter der Creative Commons Namensnennung 4.0 International Lizenz veröffentlicht, welche die Nutzung, Vervielfältigung, Bearbeitung, Verbreitung und Wiedergabe in jeglichem Medium und Format erlaubt, sofern Sie den/die ursprünglichen Autor(en) und die Quelle ordnungsgemäß nennen, einen Link zur Creative Commons Lizenz beifügen und angeben, ob Änderungen vorgenommen wurden.

Die in diesem Artikel enthaltenen Bilder und sonstiges Drittmaterial unterliegen ebenfalls der genannten Creative Commons Lizenz, sofern sich aus der Abbildungslegende nichts anderes ergibt. Sofern das betreffende Material nicht unter der genannten Creative Commons Lizenz steht und die betreffende Handlung nicht nach gesetzlichen Vorschriften erlaubt ist, ist für die oben aufgeführten Weiterverwendungen des Materials die Einwilligung des jeweiligen Rechteinhabers einzuholen.

Weitere Details zur Lizenz entnehmen Sie bitte der Lizenzinformation auf http://creativecommons.org/licenses/by/4.0/deed.de.

\section{Literatur}

1. Girmscheid, G.; Garmisch, T.; Klein, T.; Meinlschmidt, A.: Versinterung von Tunneldrainagen - Mechanismen der Versinterungsentstehung, Bauingenieur, 78 (2003), S. 292-300

2. Dietzel, M.; Rinder, T.; Niedermayr, A.; Mittermayr, F.; Leis, A.; Klammer, D.; Köhler, S.; Reichl, P.: Ursachen und Mechanismen der Ver- 
sinterung von Tunneldrainagen, BHM Berg- und Hüttenmännische Monatshefte, 153 (2008), Nr. 10, S. 369-372

3. Eichinger, S.; Leis, A.; Boch, R.; Seywald, C.; Dietzel, M.: Assessment and formation mechanisms of scale deposits in tunnels of the ÖBBInfrastruktur AG - A subproject of the Task Force Drainage // Bewertung von Sinter und deren Bildungsbedingungen in Tunnelbauwerken der ÖBB-Infrastruktur AG - Ein Teilprojekt der Task Force Drainage, Geomechanics and Tunnelling, 13 (2020), Nr. 3, S. 273-285

4. Schachinger, T.; Sperger, L.; Heissenberger, R.; Wagner, O. K.: Task Force Drainage (TFD)-The project for life after, Geomechanics and Tunnelling 10 (2017), Nr. 6, S. 779-787

5. Arbeiter, F.; Eichinger, S.; Rieß, G.; Schachinger, T.; Boch, R.; Wenighofer, R.; Galler, R.; Hausberger, A.; Strobl, E.; Stur, M.; Saliger, F.; Steiner, M.; Dietzel, M.; Pinter, G.: Optimierte Polymer-Rohrwerkstoffe für effiziente Drainagesysteme in Tunnelbauwerken - PolyDrain, BHM Berg- und Hüttenmännische Monatshefte, 164 (2019), Nr. 12, S. 545-551

6. Österreichische Vereinigung für Beton- und Bautechnik: Richtlinie Tunnelentwässerung, Wien, 2010

7. Arbeiter, F.; Eichinger, S.; Rieß, G.; Schachinger, T.; Boch, R.; Wenighofer, R.; Galler, R.; Hausberger, A.; Strobl, E.; Stur, M.; Saliger, F.; Steiner, M.; Dietzel, M.; Pinter, G.: Optimierte Polymer-Rohrwerkstoffe für effiziente Drainagesysteme in Tunnelbauwerken - Poly-
Drain Teil II, BHM Berg- und Hüttenmännische Monatshefte, 165 (2020), Nr. 12, S. 623-630

8. Sachverständigenbüro für Boden + Wasser $\mathrm{GmbH}$ : Drainagemonitoring-FactSheet FFG, https://projekte.ffg.at/projekt/2738403/pdf. Zugegriffen: 02 Sep 2021

9. Schachinger, T.; Arbeiter, F. J.; Eichinger, S.; Saliger, F.: Research on pipe materials for tunnel drainage by the ÖBB Task Force Drainage, Geomechanics and Tunnelling, 12 (2019), Nr. 5, S. 467-471

10. Austrian Standards: Klassifizierung und Eigenschaften von Techniken für die Renovierung, Reparatur und Erneuerung von Abwasserkanälen und -leitungen, Wien, ÖNORM EN 15885 (2019)

11. Österreichische Vereinigung für grabenlosen Leitungsbau: Grabenlose Bauverfahren - eine konkurrenzlose Alternative, https://www. grabenlos.at/de/grabenlose-technologie.html (13.07.2021)

12. Montanuniversität Leoben: DrainRepair: Adaptierung von Sanierungsmethoden für Drainagerohre im Tunnelbau, https://projekte. ffg.at/projekt/3791286 (13.07.2021)

Hinweis des Verlags. Der Verlag bleibt in Hinblick auf geografische Zuordnungen und Gebietsbezeichnungen in veröffentlichten Karten und Institutsadressen neutral. 